\title{
The Need for the Resultative Network
}

\author{
CRISTIANO BROCCIAS \\ University of Pavia
}

\section{Introduction ${ }^{1}$}

In this paper I will argue that in English there are various resultative constructions and that such constructions can be given a network description in the sense of Cognitive Grammar (Langacker 1987, 1991) ${ }^{2}$. First, I will discuss the 'traditional' definition of resultative phrase (cf. Levin 1993) and I will show that such a definition is unsatisfactory. Consequently, I will propose a new definition of resultative construction which implies the existence of a resultative network. In the last part of my paper, I will discuss the resultative network in more detail by focussing on its two main variants, the Force Change Schema and the Event Change Schema.

\section{What is a resultative phrase?}

The term resultative phrase usually refers to "an XP which describes the state achieved by the referent of the noun phrase it is predicated of as a result of the action named by the verb" (Levin 1993: 101). A resultative construction (RC) is the construction in which the XP and the related verb appear (e.g. John hammered the metal flat). It is argued (Levin and Rappaport Hovav 1995, among others) that XP can be predicated only of direct objects (the so-called 'direct object restriction' (DOR for short)), as shown in (1) (from Levin 1993):

(1) * Polly cooked the cookies dirty. (intended meaning: Polly became dirty by cooking the cookies)

Sentences like (2)

(2) The river froze solid.

\footnotetext{
${ }^{1}$ I would like to thank Gabriele Azzaro, Chiara Frigeni, Wilhelm Geuder, Ian Harvey, Ingrid Kaufmann, and Geoffrey Leech for their help.

${ }^{2}$ In what follows, I will assume some familiarity on the part of the reader with the basic tenets of Cognitive Grammar.
} 
are not counterexamples to the DOR, although the adjective solid is predicated of the subject (the river), because, within generative approaches, the subject of unaccusative verbs like freeze is treated as an underlying object and, within semantically-oriented accounts, 'object' corresponds to the proto-role 'patient' (see Van Valin 1990, Goldberg 1995), as is the case of the river in (2).

In what follows, I will try to show that linguistic data are much more complex than the (causal) definition of resultative phrase and the DOR imply and, consequently, that we need to introduce the notion of 'resultative network' in order to appreciate the interrelatedness of various linguistic phenomena. In other words, there exists a host of constructions that may be called 'resultative' (although this term will be used in a more general fashion than above) and such constructions can be given a network description.

\section{Some problems}

\subsection{States and positions}

Levin's definition excludes changes of position. Cross-linguistically, however, there seems to exist a correlation (see Slobin 1996) between directed uses of motion (and, perhaps, sound emission) verbs (which take positional PPs) and the availability of RCs. Indeed, some analysts regard RCs as a metaphorical extension of directed uses of motion verbs (cf. Goldberg 1995), while others don't distinguish between the two (cf. Tortora 1998). It can also be shown (Broccias 2000) that the constraints both the (transitive variants of the) RC and the directed uses of motion verbs are subject to (see Goldberg 1995) are similar, in that they evoke a force dynamics scenario (in the sense of Langacker 1991). Further, the distinction between 'state' and 'position' is sometimes a matter of degree. The PPs in (3) denote spatial configurations that do not describe actual spatial positions but must be interpreted metaphorically as states ('I was angry' in (3a) and 'The soles were worn out' in (3b)).

a. He drove me up the wall.

b. We ran the soles off our shoes.

\subsection{Non-causal resultant states}

Levin's definition implies the existence of a causal relation between the action denoted by the verb and the state expressed by the XP. Causality prevents a sentence like (4) from being categorized as an RC, despite the fact that the adjective describes a state (that of the door being shut) and that shut can appear in other 'true' (i.e. causal) RCs, as in (5).

(4) The door hissed shut.

(5) He pushed the door shut.

Analogously, (6) shouldn't be categorized as an RC because no causal relation exists between the event expressed by the verb and the PP. 
(6) She drank vodka well into her twilight years.

We note however that (6) contains a motion preposition (into), as is the case with other RCs, has some kind of resultative meaning (the PP denotes an endpoint, corresponding to the subject's referent being in her twilight years) and its existence is compatible with the schemas postulated within the resultative network (see below). Interestingly, the PP in (6) is also subject-oriented (i.e., it is predicated of the subject). This leads us to the problem addressed in the next section.

\subsection{Subject-orientation}

As has been observed by Verspoor (1997), some RCs (in the sense of Levin 1993) contain subject-oriented XPs, thus violating the DOR. We can distinguish three cases.

Some examples show a lack of a (unidirectional) energy flow from subject to object (in the sense of Langacker 1991), as in (7) ((7a) from Verspoor 1997).

a. I love you to distraction.

b. I'll fight (it) to the death.

In (7a) there is no (necessary) energy flow because the object may be unaffected by the subject's feelings (i.e., the object may be unaware of them). In (7b), fighting involves an energy flow from subject to object, but also one from object to subject: the object is in some way responsible for the death of the subject ${ }^{3}$.

A second group of examples involves verbs (of impact) that do imply a unidirectional energy flow from subject to object but also contain a PP whose complement stands in a (more or less literal) body-part relation with the constructional object, as in (8):
a. The sea air slapped me in the face.
b. It touched/shocked us to the core.
c. It cut me to the heart.

The last group of examples (see (9), from Verspoor 1997) concerns those cases in which verbs of movement (or construable as such, like play in (9d)) take both a nonmanipulable object and a PP which, adopting Langacker's terminology, realizes a salient part of the verb's base.

\footnotetext{
${ }^{3}$ Interestingly, subject-orientation seems to obtain with the PP to the death, whereas the PP to death is usually object-oriented.
} 

a. We followed the star out of Bethlehem.
b. The sailors rode a breeze clear of the rocks.
c. John danced mazurkas across the room.
d. The children played leapfrog across the park.

Before concluding this section, I would like to cite two other examples, (10) and (11), which I will not discuss further for reasons of space but which provide additional evidence for the complexity of the notion of RC.

(10) She rode the horse to town.

(11) a. I gave him a hand outside (i.e., to go outside).

b. His window was thrown wide to the sun.

(10) shows that in some cases a PP can be predicated of both the subject and the object: both the subject's and the object's referent can be said to be in town once the action denoted by the verb is over. (11a) demonstrates that an RC can contain two objects (him and $a$ hand) and (11b) shows that two resultative phrases (wide and to the sun) can sometimes cooccur (see also Goldberg 1991).

\subsection{Interim conclusions}

The examples discussed so far have shown that the notion of RC is much more complex than is usually assumed. Some constructions can be considered resultative and yet they lack a causal relation between the verb and the final state/position expressed by the XP. Other examples indicate that subject orientation does occur, contrary to what is implied by the DOR. I will argue that a neater picture emerges if we introduce the notion of 'resultative network'.

\section{The resultative network}

\subsection{A revised (non-causal) definition}

In order to capture the similarities among the examples discussed in the previous section, we could interpret the term 'resultative phrase' in a more general fashion as referring to either a state or position achieved by an entity $a$ involved in some event $\mathrm{E}$, provided that $a$ is spelled out as a constructional argument. This definition neither requires the existence of a causal relation between the constructional verb and the XP, thus allowing for the 'problematic' cases discussed in 2.2, nor makes any categorial distinction between states and positions (versus Goldberg 1995). All the constructions that satisfy this definition can be said to make up a 'resultative network', in the sense that (a) we can group some of them together as realizations of a particular schema because of their common features and (b) such groups are related to one another.

\subsection{A sublexical definition}

It should be stressed that even the revised definition of the term 'resultative' proposed in 3.1. does not encompass all possible resultative cases. In fact, it 


\section{The Need for the Resultative Network}

explicitly requires the resultative phrase to be predicated of a phonologically realized argument. However, there are some cases that resemble resultative constructions and yet do not satisfy the above definition. Consider (12):
a. The man was sobbing into his hands.
b. She shouted up into the trees.
c. He fired a machine gun into the supermarket.

They all contain verbs that can be described as 'verbs of emission'. With such verbs the PP could be said to refer to the verbal event (see O'Dowd 1998:112). For example, it is the sobbing in (12a) that goes into the pillow, not the subject's referent. But we also note that a verb like shout allows the addition of a direct object (one can shout something up into the trees) of which, then, the PP can be taken to be predicated. Consequently, the other examples in (12) could be treated in a similar fashion by postulating the existence of a 'null' object of which the PP is predicated. However, (12c) shows that such a move is sometimes untenable; the $\mathrm{PP}$ in (12c) clearly refers to the firing event, not to the gun. In my view the two alternatives (predication over events vs. predication over objects) amount to different paraphrases of the same semantic representations, which are given in (13):

a. see (12a-b)

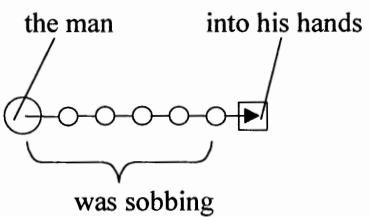

b. see $(12 \mathrm{c})$

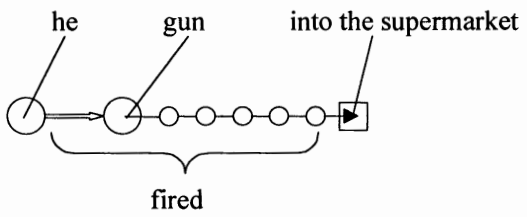

The schema in (13a) is relevant for the examples (12a-b), the one in (13b) for (12c). A verb of emission is represented through a series of small circles-they represent what is emitted (e.g. a series of sounds) - originating from an entity (the trajector, the leftmost circle). The constructions in (12) also specify a telic path, which has been represented in (13) as the arrow connecting the smaller circles plus the square box towards which the small circles move. (13a) captures the intuition that the resultative phrase seems to refer to a null object (i.e., the emitted substance) and the fact that what is emitted is produced by the subject. The schema in (13b) shows the semantic pole of (12c). The verb lexicalizes both the force (the double arrow) exerted by the subject (the leftmost circle) onto the object (the big circle on the right, corresponding to the gun in (12c)) and what is emitted by the gun (the smaller circles). In analogy with (13a), it is the emitted substance that moves towards the location represented as the square box. This explains why the PP in (12c) is not object-oriented. 
If we regard the examples in (12) as 'resultatives', we should then interpret this term as also referring to sublexical entities (i.e. entities whose existence can be postulated at the semantic pole but which are not realized independently of the verb at the phonological pole):

\section{(14) definition of resultative phrase}

A phrase is said to be resultative if it refers to a state or position achieved by an entity $a$ involved in an event $\mathrm{E}$, provided that $a$ can be postulated at the semantic pole of the relevant construction.

\subsection{The Force Change Schema and the Event Change Schema}

In what follows I will concentrate on that portion of the resultative network that is relevant for the cases where the resultative phrase refers to a lexical entity.

I propose that within the resultative network we recognize two fundamental schemas, the Force Change Schema (FCS) and the Event Change Schema (ECS), represented in (15a) and (15b), respectively.

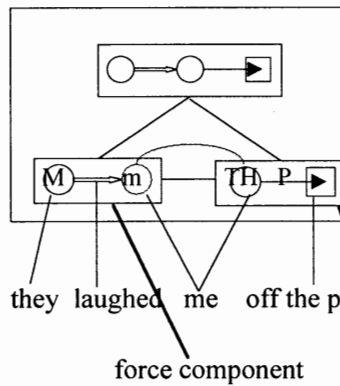

$\mathrm{M}=$ Manipulator, $\mathrm{m}=$ manipulee, $\mathrm{TH}=$ theme, $\mathrm{P}=$ path, $\mathrm{tr}=$ trajector, $\mathrm{E}=$ event b. ECS

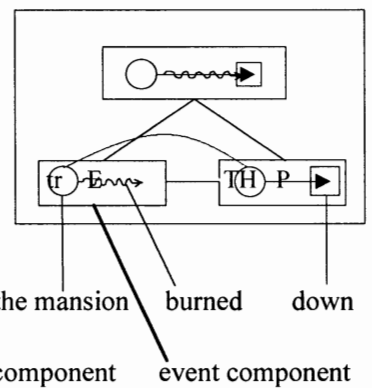

The semantic pole of the FCS describes a forcible interaction (see the force component in (15a)) between a manipulator $(M)$ and a manipulee $(m)$, resulting in the change of state or position of the latter (see the change component in (15b)). In more detail, the force component ( $\mathrm{F}$ or $\mathrm{F}$ component, for short) comprises an $M$ which exerts a force (represented by the arrow) onto an $m$, the dotted circle. The change component ( $\mathrm{C}$ or $\mathrm{C}$ component, for short) contains an entity, which I will refer to as theme ( $T H$ for short), which moves literally or metaphorically (i.e. it is subject to a change of state/position), as indicated by the arrow $P$ (path), towards a location (whether literal or metaphorical), represented as a square. The construction requires the identity of both $m$ and $T H$ as indicated by the dotted line connecting the two. The $m$ circle is dotted to signify that the entity acted upon is not necessarily selected by the constructional verb (as in They laughed him off the podium). The integration of $\mathrm{F}$ and $\mathrm{C}$ results in the structure depicted as the upper 
box; it shows that $M$ is responsible for the change of state/position of $m$. The phonological pole of the FCS (not represented in (15)) requires the C component to be expressed by an XP. XP can correspond to an AP, as in She shot him dead, a $\mathrm{PP}$, as in He rocked her to sleep, an NP, as in They appointed him president, or a combination of them, as in (11b).

The FCS expresses the commonality of those cases which are usually called resultative, that is those cases which display both a causality link between the verb and the resultant state (see 2.2.) and object orientation (cf. the DOR).

Let us now examine the ECS (see (15b)). The ECS also results from the integration of two components, namely the event component ( $\mathrm{E}$ or $\mathrm{E}$ component, for short) and the change component, identical to the one within the FCS. The former shows an entity, a trajector (tr), as being involved in an event $\mathrm{E}$. The $\mathrm{E}$ component and the $\mathrm{C}$ component are integrated into each other by imposing the identity of $t r$ and $T H$, as indicated by the dotted line connecting the two. We obtain a complex event, represented as the upper box in (15b), which specifies that the $\mathrm{E}$ component and the $\mathrm{C}$ component are projected onto each other, as will be explained below.

The ECS is relevant, among other things, for 'traditional' intransitive resultatives, such as The river froze solid, as shown in (15b).

Before illustrating the ECS in more detail, I would like to observe that some FCS examples contain causative verbs, so that they describe the activity carried out by the constructional object rather than the constructional subject, as in (16).

I rattled out a box of candles.

In order to capture this fact, we could posit a variant of the FCS where the C component corresponds to the ECS, as in (17):

(17)

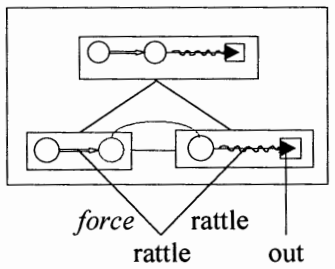

(17) shows that the transitive verb rattle is both associated with the F and the $\mathrm{C}$ components. In other words, the sound emission cooccurs with the movement of the box (as indicated by the $\mathrm{C}=\mathrm{ECS}$ component, corresponding to the construction where intransitive rattle occurs) and such cooccurrence of events is due to a manipulator (hence the association of the verb with the force component). 


\subsection{Two differences between the FCS and the ECS}

Given the systemic nature of language (i.e. language as a network), the ECS obviously does not subsume the cases mentioned in connection with the FCS (i.e., 'true' resultative examples). There are two differences between the FCS and the ECS and they concern the notions of (a) ordering of the subcomponents and (b) force.

\subsubsection{Ordering}

Whereas the FCS imposes a fixed temporal sequencing and a causal ordering for $\mathrm{F}$ and $\mathrm{C}$ ( $\mathrm{F}$ precedes and causes $\mathrm{C}$ ), the ECS doesn't because it requires the E and $\mathrm{C}$ components to be projected onto each other (as indicated in the upper box within the $\left.\mathrm{ECS}^{4}\right)$. This allows us to use the bottom boxes to express, when relevant (see below), the relation of causality between $\mathrm{C}$ and $\mathrm{E}$. In other words, $\mathrm{C}$ and $\mathrm{E}$ are cooccurrent (upper box), but one (usually) causes the other (linear order of the boxes below).

(15b) illustrates the case in which $\mathrm{E}$ causes $\mathrm{C}$. Note that examples like The river froze solid (see (18a)) are analogous to (15b), the only difference being the association of freeze with both the $\mathrm{E}$ and $\mathrm{C}$ components; solid simply spells out the resultant state (cf. the notion of imposition of an ADC construal in Broccias 2000). (18b) illustrates the case in which $C$ causes $E$ (example from Goldberg 1995).

(18)

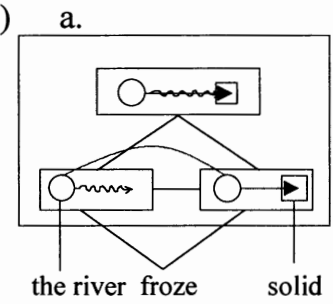

b.

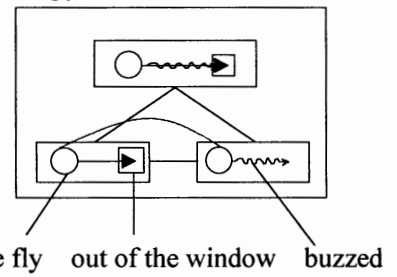

(i.e., The fly buzzed out of the window)

(6), represented diagrammatically in (19), shows that the notion of causality is not always relevant for the ECS (as suggested by the lack of an ordering for $\mathrm{E}$ and $\mathrm{C}$ in (19)). What is crucial here is simply the cooccurrence of the $\mathrm{E}$ and $\mathrm{C}$ components.

\footnotetext{
${ }^{4}$ A similar idea, namely that of necessary temporal dependence, has been developed by Rappaport Hovav and Levin (1999). However, Broccias (2000) argues that a network approach such as the one proposed here correctly accounts for the impossibility of examples like ${ }^{*} I$ washed clean (vs. I washed myself clean), which should be acceptable within Rappaport Hovav and Levins's model.
} 


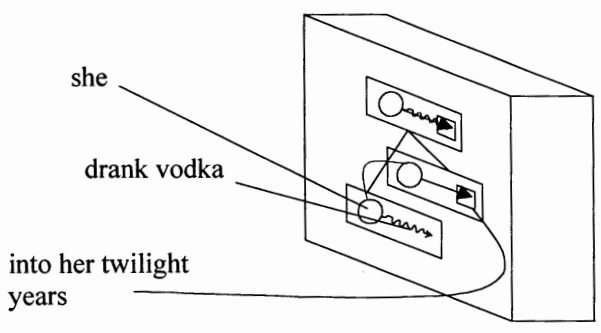

\subsubsection{Force}

The ECS cannot be taken simply as a schema which groups together those cases where a force component is lacking (as is the case with intransitive resultatives). Example (6) contains a verb which can be conceptualized as implying a unidirectional energy flow (drink), and yet (6) is not associated with the FCS because no causal relation exists between the action denoted by the verb and the final state predicated by the PP. The examples in (7) and (9) cannot be linked to the FCS either, because they lack a necessary unidirectional energy flow from subject to object (see 2.3). On the other hand, they can be associated to the ECS because it is the $\mathrm{E}$ component (e.g., loving, following, playing) that determines the $\mathrm{C}$ component and the two can be said to be projected onto each other: the actions of loving, following, playing, etc. are construed as paths leading to the state/position denoted by the resultative phrase. Therefore, we conclude that, when the notion of causality is relevant, the ECS is also compatible with a force dynamics scenario, provided that this is not identical to that evoked by the FCS.

A compact and simplified representation for (7a), which I will adopt here also for (9a-b), and (7b) is offered in (20a) and (20b) respectively. In (20a), the simple downward arrow expresses the intuition that the subject's referent engages in a non-forcible relation with the object (i.e., the object is not affected). A force arrow connects the landmark to the trajector because the object's referent is conceptualized as being responsible for the change of position/state of the subject.

In (20b), the double headed arrow is meant to indicate that both the subject exerts a force onto the object and that, as in (20a), the object is responsible for the subject's change of state. This contrasts with the instantiations of the FCS, where the single headed arrow visualizes the fact that the energy flow is unidirectional (from the trajector to the landmark). (20b) also contains a dotted landmark to indicate that the object is optional.

The semantic pole for $(9 \mathrm{c}-\mathrm{d})$ is given in $(20 \mathrm{c})$. The $\mathrm{E}$ component is represented through three component states (the circles connected to the squares). The $\mathrm{C}$ component is shown as the arrow connecting the three circles. Further, Langacker (1991: 363) notes that "the component states of a process constitute a set of interconnected entities and thus implicitly define an abstract region". I 
propose that constructions like $(9 \mathrm{c}-\mathrm{d})$ profile such a region (represented as the circle encompassing the component states of the relevant process), which corresponds to the nonmanipulable object in the relevant construction.
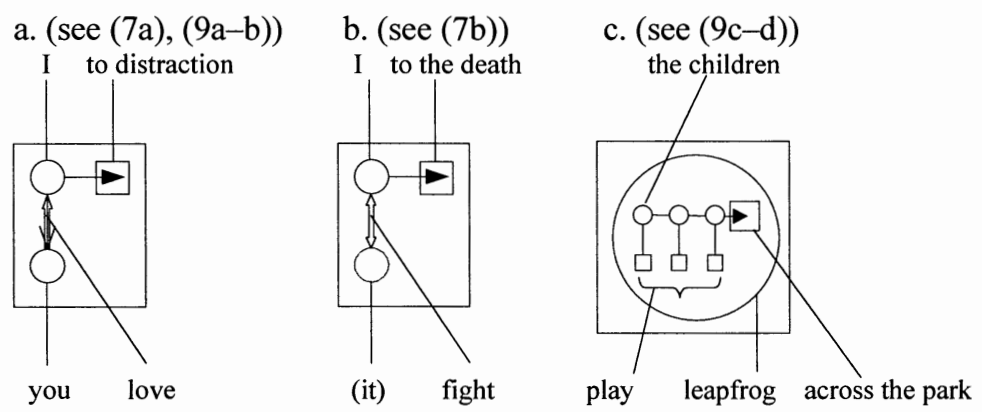

We still have to consider the body-part examples in (8a-c). They can be analyzed as instantiations of a schema, the Body-Part Schema (BPS, see (21)), which shares features of both the FCS and the ECS. As in the former, the BPS evokes a force dynamics scenario based on a unidirectional energy flow from subject to object (see the F component in (21)), but unlike the FCS it requires the identity of the $M$ and the $T H$ (i.e., we have subject-orientation for the XP as with the ECS); this is indicated by the dotted line connecting the two. Furthermore, the $m$ stands in a part-whole relation with the resultant location (the square within the $\mathrm{C}$ component) as indicated by the line connecting the two in the lower boxes and by drawing the square box (the part) in the circle (the whole) in the upper box. Finally, the force component and the change component are projected onto each other (see the upper box in (21)) in analogy with what happens with the $\mathrm{E}$ and the $\mathrm{C}$ components in the ECS.

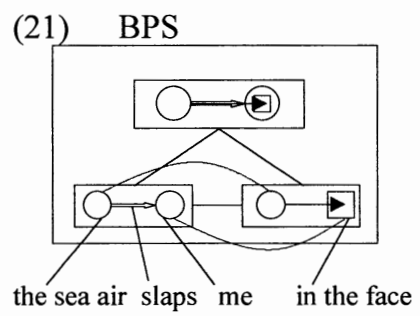

\section{Conclusions}

In this paper I have shown that (a) the definition of resultative phrase adopted by Levin (1993) is too restrictive because it excludes various cases which are intuitively resultative but do not fall under her definition and (b) that the DOR cannot be maintained. I have suggested that a neater picture emerges if we 


\section{The Need for the Resultative Network}

introduce the notion of 'resultative network'. Within it, what are usually described as resultative constructions are instantiations of two units, the Force Change Schema, FCS, (which is relevant for 'usual' transitive cases such as They laughed me off the podium) and the Event Change Schema, ECS, (which is relevant for 'usual' intransitive cases like The river froze solid). I have also shown that the examples that do not fit Levin's definition can be analyzed as instantiations of the ECS or instantiations of schemas, like the BPS, that share features of both the FCS and the ECS. The resultative network can be given a compact representation as in the following diagram:

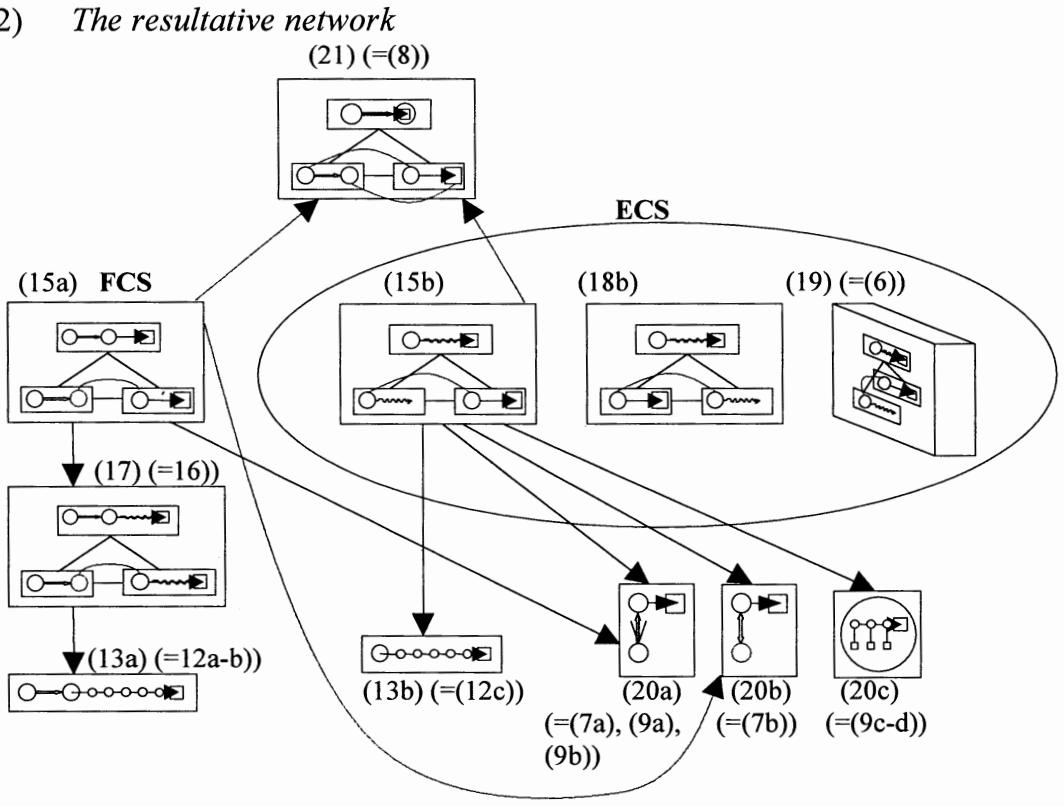

\section{References}

Broccias, Cristiano. 2000. The resultative construction as an instantiation of the affected directional construction. Paper presented at the 22nd Meeting of the Deutsche Gesellschaft für Sprachwissenschaft. Marburg, 1-3.03.2000.

Goldberg, Adele. 1991. It can't go up the chimney down: Paths and the English resultative. Berkeley Linguistics Society 17: 368-378. 


\section{Cristiano Broccias}

Goldberg, Adele. 1995. Constructions: A Construction Grammar Approach to Argument Structure. Chicago and London: The University of Chicago Press.

Langacker, Ronald. 1987. Foundations of Cognitive Grammar. Volume I. Theoretical Prerequisites. Stanford, CA: Stanford University Press.

Langacker, Ronald. 1991. Foundations of Cognitive Grammar. Volume II. Descriptive Applications. Stanford, CA: Stanford University Press.

Levin, Beth. 1993. English Verb Classes and Alternations. Chicago: The University of Chicago Press.

Levin, Beth and Malka Rappaport Hovav. 1995. Unaccusativity: At the SyntaxLexical Semantics Interface. Cambridge, MA: MIT Press.

O'Dowd, Elizabeth. 1998. Prepositions and Particles in English: A Discoursefunctional Account. Oxford: Oxford University Press.

Rappaport Hovav, Malka \& Beth Levin. 1999. Two types of compositionally derived events. Ms., Bar Ilan University \& Northwestern University.

Slobin, Dan. 1996. Two ways to travel: Verbs of motion in English and Spanish. In Masayoshi Shibatani and Sandra Thompson (eds.) Grammatical Constructions. Their Form and Meaning. Oxford: Clarendon Press.

Tortora, Christina. 1998. Verbs of inherently directed motion are compatible with resultative phrases. Linguistic Inquiry 29: 338-345.

Van Valin, Robert. 1990. Semantic parameters of split intransitivity. Language 66: $221-260$.

Verspoor, Cornelia. 1997. Contextually-dependent lexical semantics. PhD dissertation, University of Edinburgh.

Cristiano Broccias

Dipartimento di Linguistica

Universita' di Pavia

Corso Strada Nuova, 65

27100 Pavia

Italy

cribroc@tin.it 